\title{
Depressed electron collector for the Gatling Gun Test Stand
}

\author{
A. Pikin, I. Ben-Zvi, X.Y. Chang, D. Gassner, \\ V. Litvinenko, O. Rahman, T. Rao, E. Riehn, \\ B. Sheehy, J. Skaritka, E. Wang, Q. Wu
}

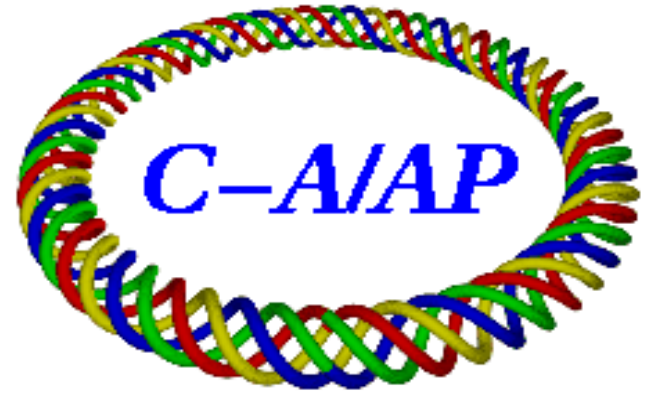

\section{Collider-Accelerator Department Brookhaven National Laboratory Upton, NY 11973}

Notice: This document has been authorized by employees of Brookhaven Science Associates, LLC under Contract No. DE-AC02-98CH10886 with the U.S. Department of Energy. The United States Government retains a nonexclusive, paid-up, irrevocable, world-wide license to publish or reproduce the published form of this document, or allow others to do so, for United States Government purposes. 


\title{
Depressed electron collector for the Gatling Gun Test Stand*
}

\author{
A.Pikin ${ }^{\dagger}$, I. Ben-Zvi, X. Y. Chang, D. Gassner, V. Litvinenko, O. Rahman, T. Rao, E. Riehn, \\ B. Sheehy, J. Skaritka, E. Wang, and Q. Wu \\ Brookhaven National Laboratory
}

\begin{abstract}
An optical solution for, and the general design of an electron collector (EC) for a Gatling gun Test Stand are described. The purposes of this development were to reduce the power load on the electron gun's high-voltage power supply, and to eliminate the back-streaming of electrons from the dump into the ultra-high-vacuum region of the beam line and the gun chamber, thereby preventing vacuum degradation. The challenge is solved by creating an effective biased "black box" on the cathode terminal for all electrons, and by assuring the vacuum separation of the EC's volume from the beam line.
\end{abstract}

\section{INTRODUCTION}

BNL is developing a future electron-ion collider, eRHIC [1-2], utilizing a polarized electron source based on a gun with multiple photocathodes (i.e., a Gatling gun) driven by IR lasers with circular polarization [3-5]. The electron bunches from individual cathodes will be directed to a common beam-line axis by a fast combiner with rotating magnetic field [6]. A stand for testing the Gatling gun that encompasses the major elements of the preinjector: an electron gun, and a combiner is under development. The electron beam itself will be used only for testing the performance of these major units. This application will allow us to decelerate the electron beam, to nearly the cathode potential at the electron collector (EC). Collecting the electron beam at a low energy reduces the gas load in the vacuum chamber. Effectively trapping all the electrons, primary, secondary, and backscattered ones, reduces the power load on the gun's highvoltage power supply, and lowers the gas load caused by the returned electrons. This design also

\footnotetext{
* Work supported under the auspices of the US Department of Energy and the National Aeronautics and Space Administration.

† pikin@bnl.gov
} 
should diminish the probability of high-voltage discharges in the gun's chamber. Considering that the quantum efficiency and the charge life time of the photocathodes depend upon the vacuum conditions in the gun chamber, implementing the depressed electron collector seems very beneficial for the gun’s overall performance.

\section{DESIGH AND OPTICAL SOLUTION}

Fig.1 is a general schematic of the Gatling gun's test stand. The depressed collector with its power supplies and the NEG pump are mounted on a separate platform that is biased by approximately $+20 \mathrm{kV}$ with respect to the cathode's potential. The purpose of having this bias power supply is to drive the electron beam's current with its energy spread back to the cathode potential. The electron current, which is dissipated in the EC, is a load on the collector bias power supply. Therefore, to assure effective electron collection, the maximum voltage of this power supply should be greater than the expected electron energy spread, and its maximum output current should be larger than the expected electron current of the beam.

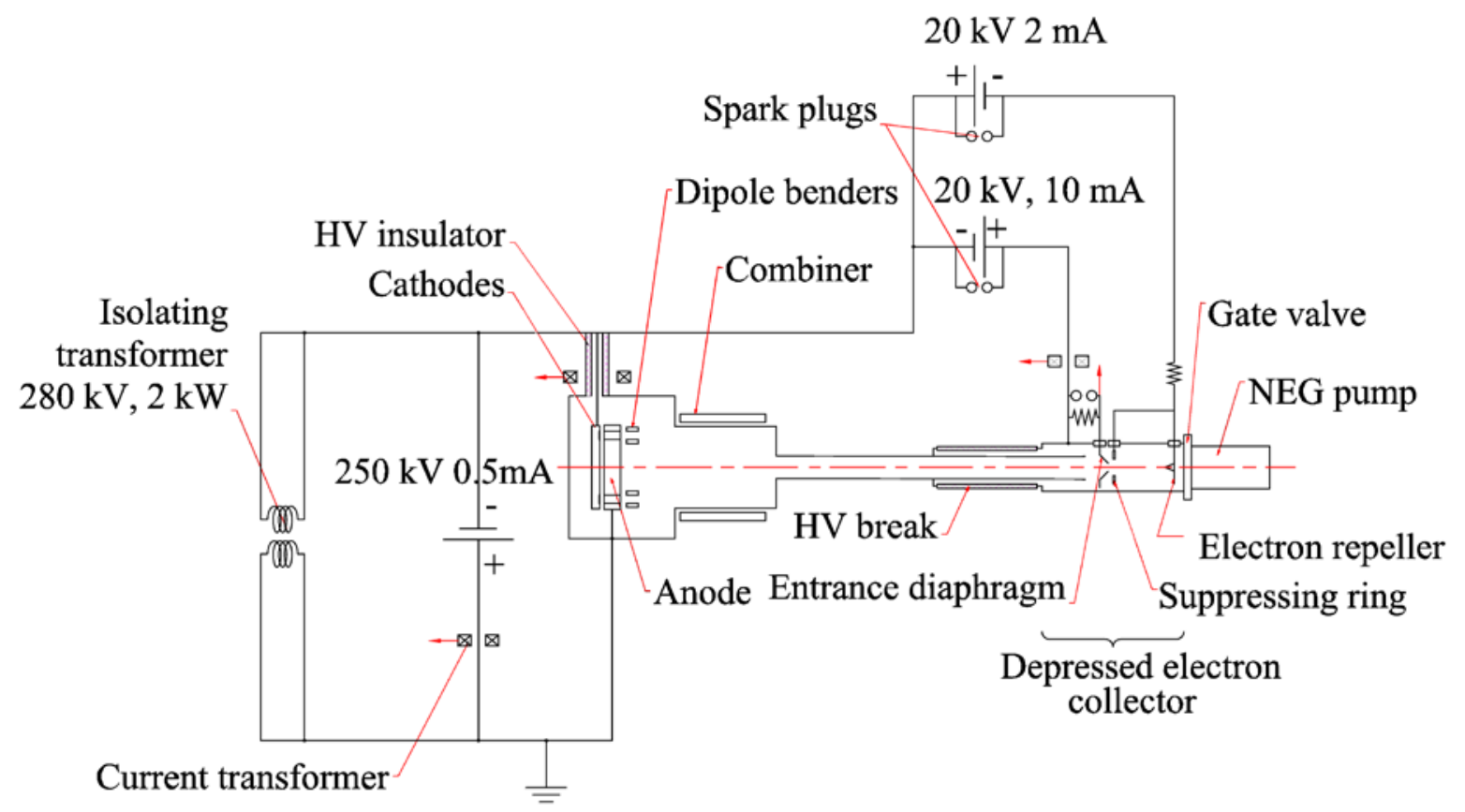

Fig. 1. Schematic of the Gatling gun test stand. 
The power supply of the electron reflecting electrodes also is located on the same cathode terminal. An insulating transformer supplies power to these power supplies and to the diagnostics units with the control electronics.

The electron collector is mounted on a high-voltage terminal of the ceramic break. The magnetic lens and the magnetic deflectors for steering the electron beam into the entrance diaphragm are located next to the high-voltage break at the ground potential. The aperture of this diaphragm was minimized such to reduce the gas flux from the EC volume into the beam line while yet accepting the full electron energy spread. To effectively reduce the returned flux of the primary, secondary, and backscattered electrons into the high-vacuum volume of beam line it is proposed to collect the primary electrons on the cylindrical wall of the electron collector. Siting the edge of the beam line's grounded tube close to the electron collector's entrance diaphragm assures the required symmetric radial divergence of the over-focused electron beam. The electric field of two reflecting electrodes controls the region of primary electron deposition.

Fig.2 illustrates the overall design of the electron collector

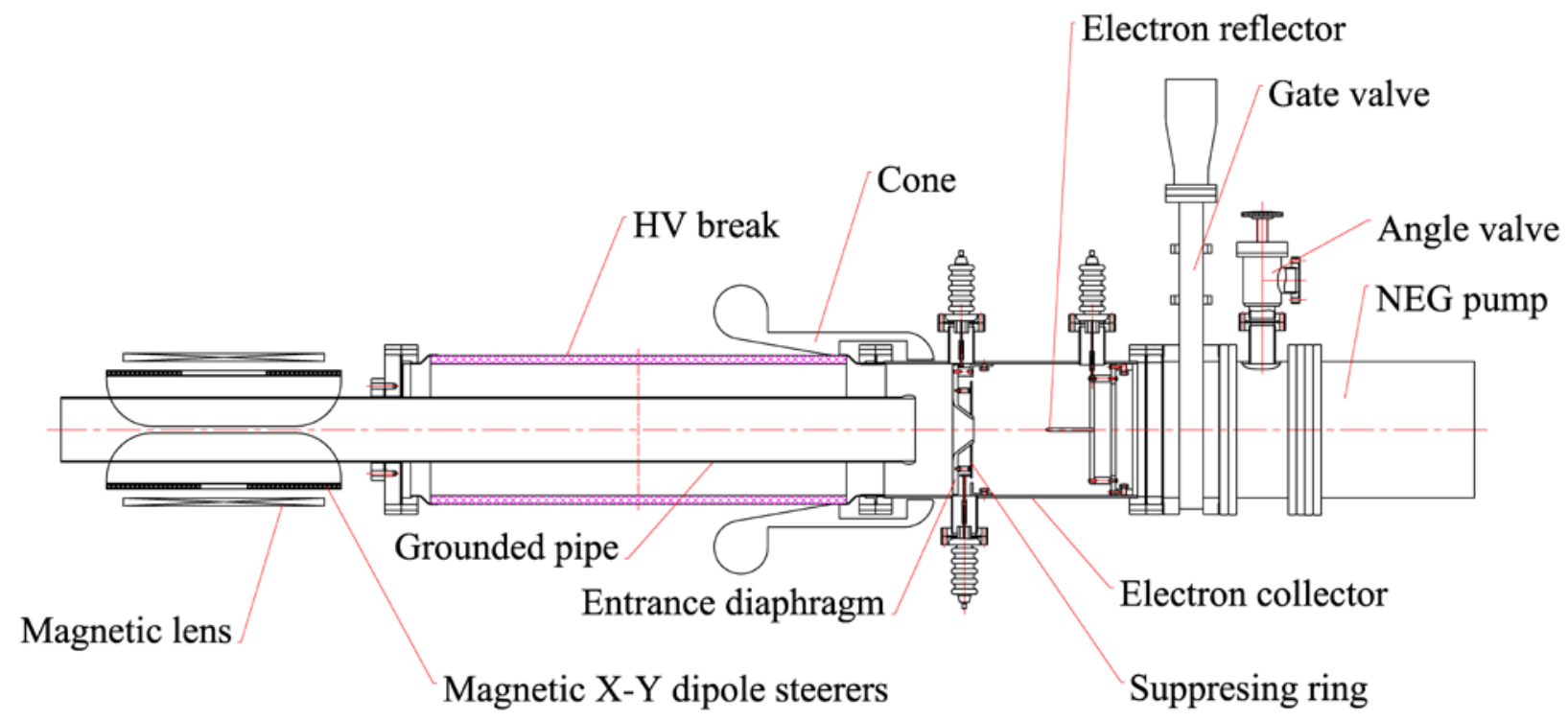

Fig. 2. Structure of the depressed electron collector assembly for the Gatling gun's test stand.

Aluminum is a preferred material for the internal wall of the collector because its backscattering coefficient is lower than that of materials containing heavier elements, like copper. The NEG 
pump, mounted behind the electron reflector, has large vacuum conductance to the electron collector's internal volume. It is equipped with a gate valve and an angle valve for nondisruptive activation.

The combination of the entrance diaphragm with its limited aperture and a NEG pump creates an efficient vacuum separation of the electron collector's volume from that of the electron gun.

\section{SIMULATION OF THE ELECTRON COLLECTOR}

\section{A. Electric field simulation}

The cathode voltage $\left(\mathrm{U}_{\text {cath }}\right)$ is defined as a potential difference between the cathode of the gun and the grounded anode. The collector's voltage $\left(\mathrm{U}_{\mathrm{col}}\right)$ is defined as a potential difference between the electron collector and the cathode of the gun. The electron reflector's electrode and suppressing ring are connected, and their potential $\left(\mathrm{U}_{\text {refl }}\right)$ also is referenced to the cathode. Electric field distribution was simulated with the ESTAT program of Field Precision [7]; Fig 3 shows the result for the area with the highest electric field.

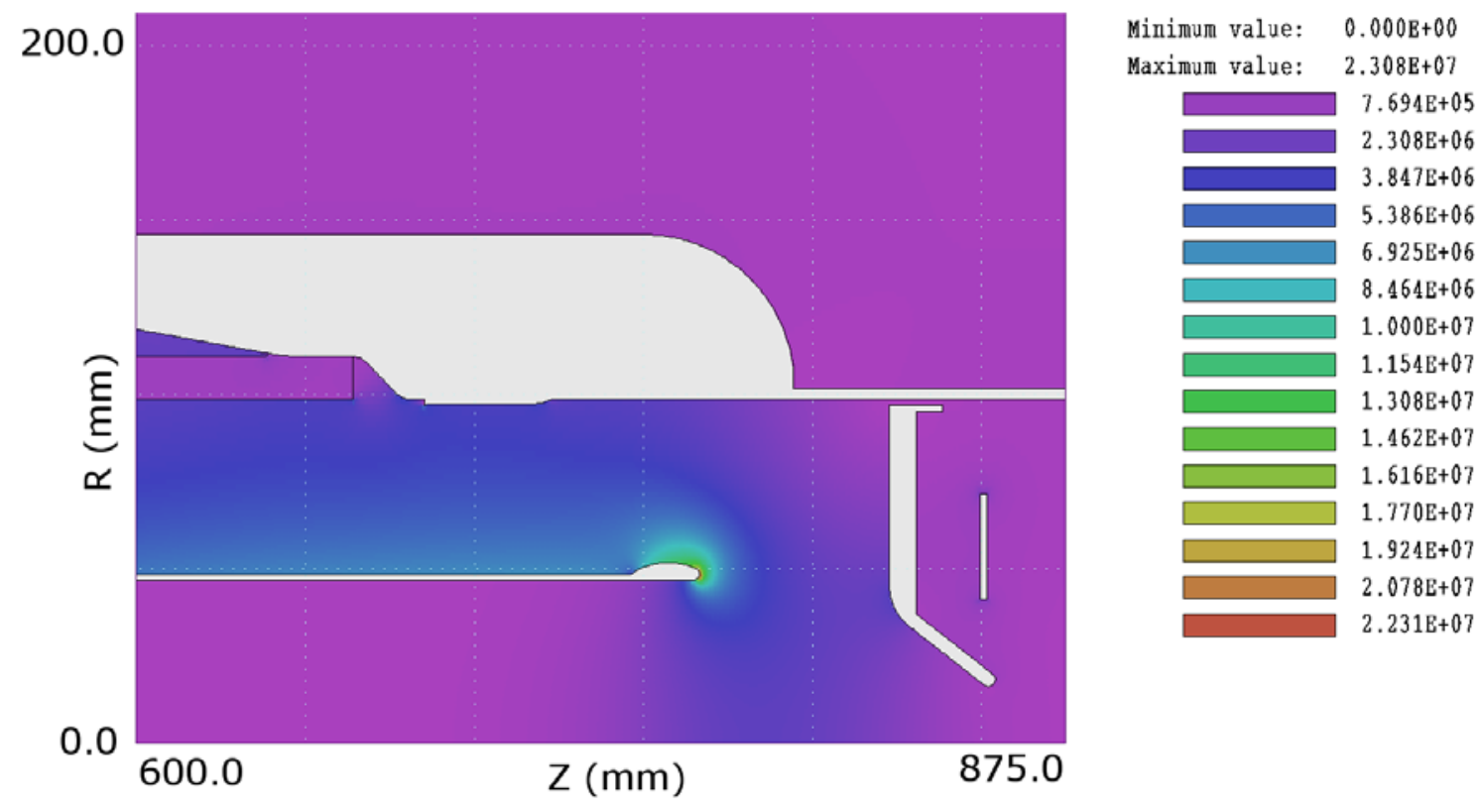

Fig. 3. The map of the distribution of the electric field in a gap between electron collector and a ground pipe. $\mathrm{U}_{\text {cath }}=250.0 \mathrm{kV}, \mathrm{U}_{\text {col }}=15.0 \mathrm{kV}$. 
On the negative electrode, the highest field is $E_{n e g}=3.7 \mathrm{kV} / \mathrm{mm}$ for the potential on the electron collector $U_{\text {col }}=235.0 \mathrm{kV}$. The maximum Kilpatrick field of $E_{\text {Kilpatrick }}=7.88 \mathrm{kV} / \mathrm{mm}$, corresponding to the safety factor of 2.1. The cone helps in distributing the electric field fairly uniformly along the ceramic break within a range of $0.2-0.68 \mathrm{kV} / \mathrm{mm}$.

\section{B. Primary electrons}

All trajectory simulations of the electron beam have been done with the TRAK program [7]. The following are the parameters of the electron beam:

- Electron current is $\mathrm{I}_{\mathrm{el}}=2.0 \mathrm{~mA}$,

- Electron energy is $\mathrm{E}_{\mathrm{el}}=250 \pm 10 \mathrm{keV}$ with a uniform energy distribution in this range,

- Initial radius of the electron beam is $r_{b}=20.0 \mathrm{~mm}$,

- Number of electron trajectories, $\mathrm{N}_{\text {traj }}=500$,

We assumed that the emittance of the electron beam was zero (a laminar beam) and that the radial distribution of the electron beam's current density was uniform. The maximum magnetic field in the magnetic lens was 44 Gs.

Fig.4 depicts the result of our preliminary simulation of the primary electron beam.

In this simulation, two central trajectories (out of 500) with total electron current of $\cdot 4.8{ }^{-8} \mathrm{~A}$ have returned back.

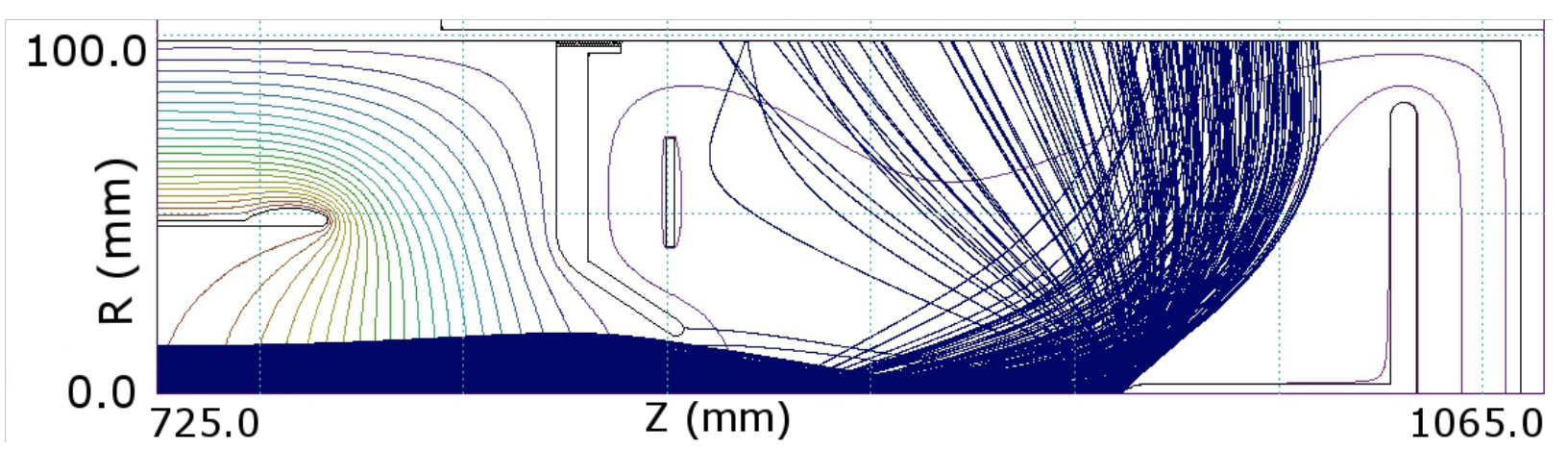

Fig. 4. Simulated transmission of the electron beam in the depressed electron collector.

$$
\mathrm{U}_{\mathrm{col}}=15.0 \mathrm{kV}, \mathrm{U}_{\mathrm{refl}}=-5 \mathrm{kV} \text {. }
$$

As expected, the returned current of the primary electron beam depends on the collector's voltage, $\mathrm{U}_{\mathrm{col}}$. 
Here, we define the efficiency of the absorption of the primary electron beam by the collector as the ratio of the absorbed primary electron current $\left(\mathrm{I}_{\mathrm{col}}\right)$ on the collector to the total initial electron current $\left(\mathrm{I}_{\text {start }}\right)$. Fig.5 illustrates the simulated dependence of the efficiency of the electron absorption on the collector voltage.

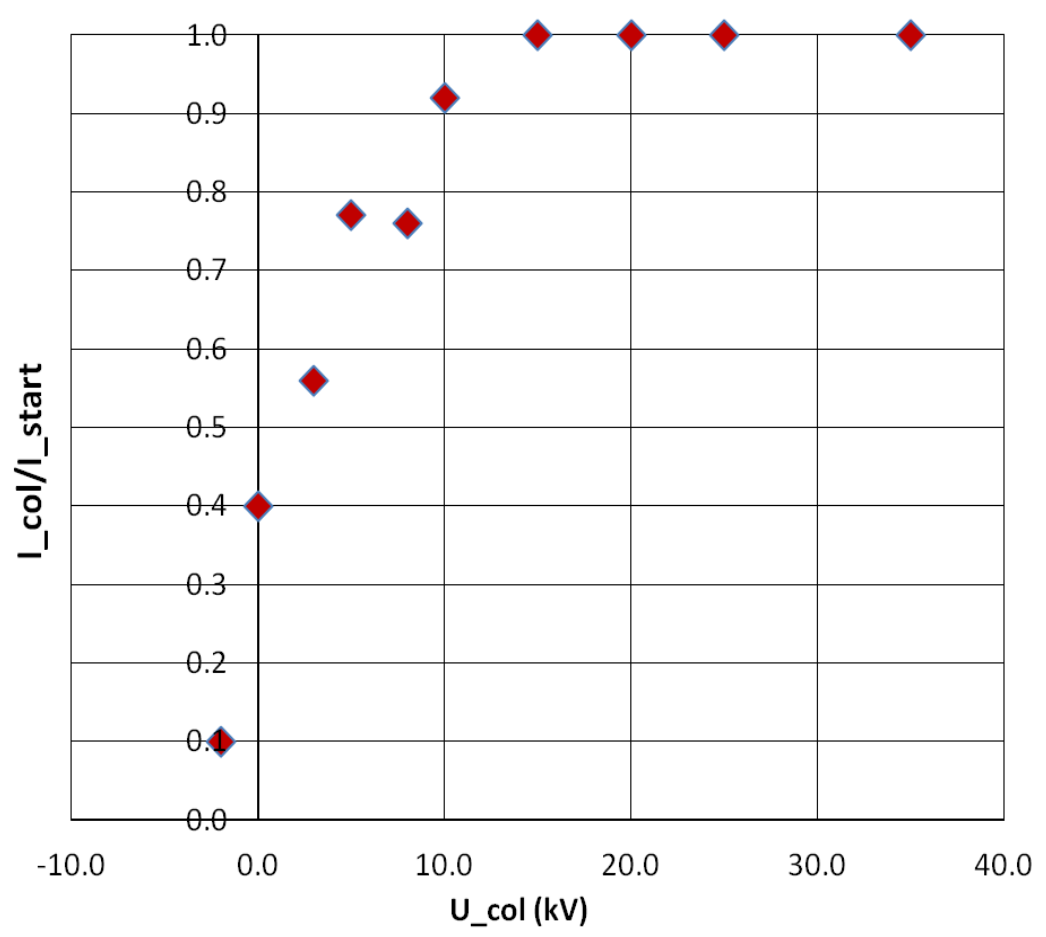

Fig. 5. Dependence of the primary electron beam's absorption efficiency on the collector's voltage for $\mathrm{U}_{\text {refl }}=-5 \mathrm{kV}$.

It is evident that with the electron collector' voltage above $15 \mathrm{kV}$, practically all primary electrons are absorbed within its volume.

\section{Backscattered electrons}

These simulations encompassed some conservative assumptions. Our aim was identifying an optical solution assuring that the secondary particles are completely blocked. In these simulations, we conservatively assumed that the energy of backscattered electrons was $90 \%$ of that of the primary electrons. The coefficient of backscattering was taken as 0.3 (for copper) for any incident angle within the energy range of the primary electrons, $E_{\text {prim }}=50 \div 20000 \mathrm{eV}$. The 
angular distribution of the emitted backscattered electrons was assumed to follow a cosine function about the normal to the metal surface. All simulated backscattered electrons start at the end of the primary electron's trajectory, so the origin of any backscattered electron has the coordinates of the striking point of the corresponding primary electron. Only the first generation of backscattered electrons was simulated and the result is shown in Fig. 6.

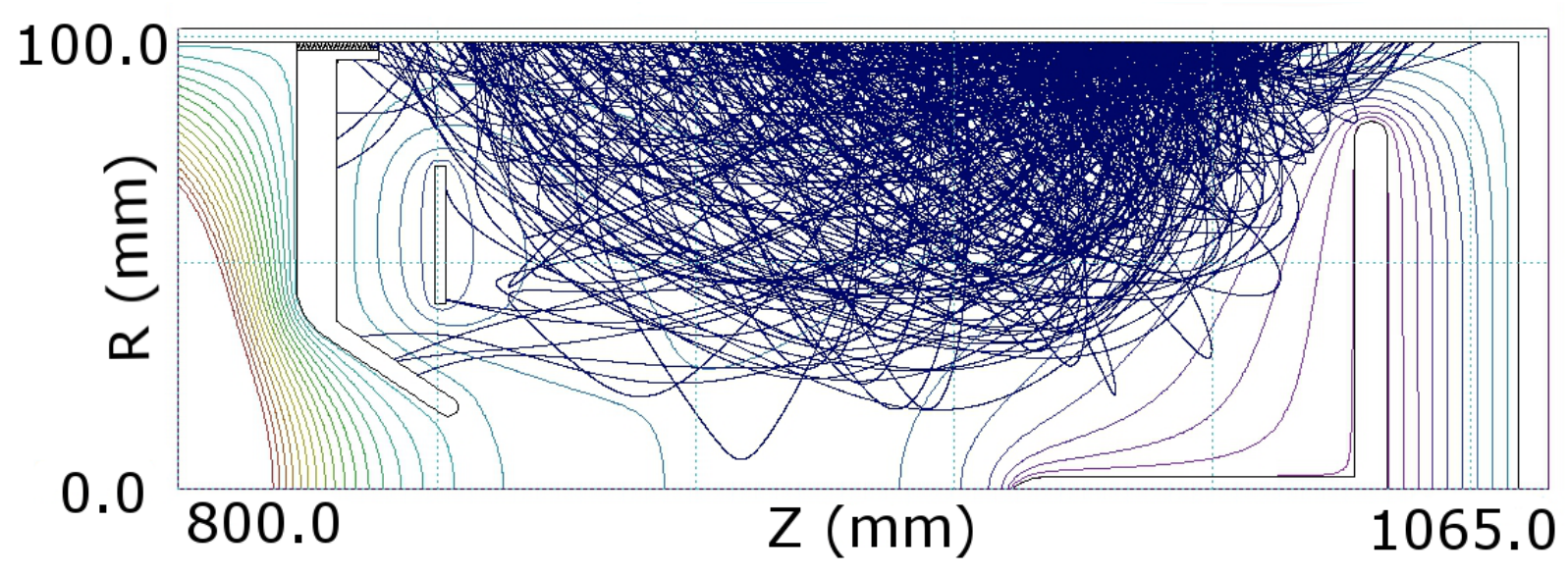

Fig. 6. Simulated trajectories of the backscattered electrons for $\mathrm{U}_{\text {cath }}=250 \mathrm{kV}, \mathrm{U}_{\mathrm{col}}=15.0 \mathrm{kV}$,

$$
\mathrm{U}_{\text {refl }}=-5 \mathrm{kV} \text {. }
$$

In this simulation, none of 500 particles passed through the entrance diaphragm into the beam line.

\section{Secondary electrons}

In this set of simulations, the energy range of the secondary electrons was within $1 \div 50 \mathrm{eV}$; we assumed a uniform energy spectrum within this range. The starting point of secondary electrons and the angular distribution was taken the same as for the backscattered electrons. The coefficient of secondary electron emission was taken as 1.5 for the entire range of primary electron energy. The result is presented in Fig. 7. 


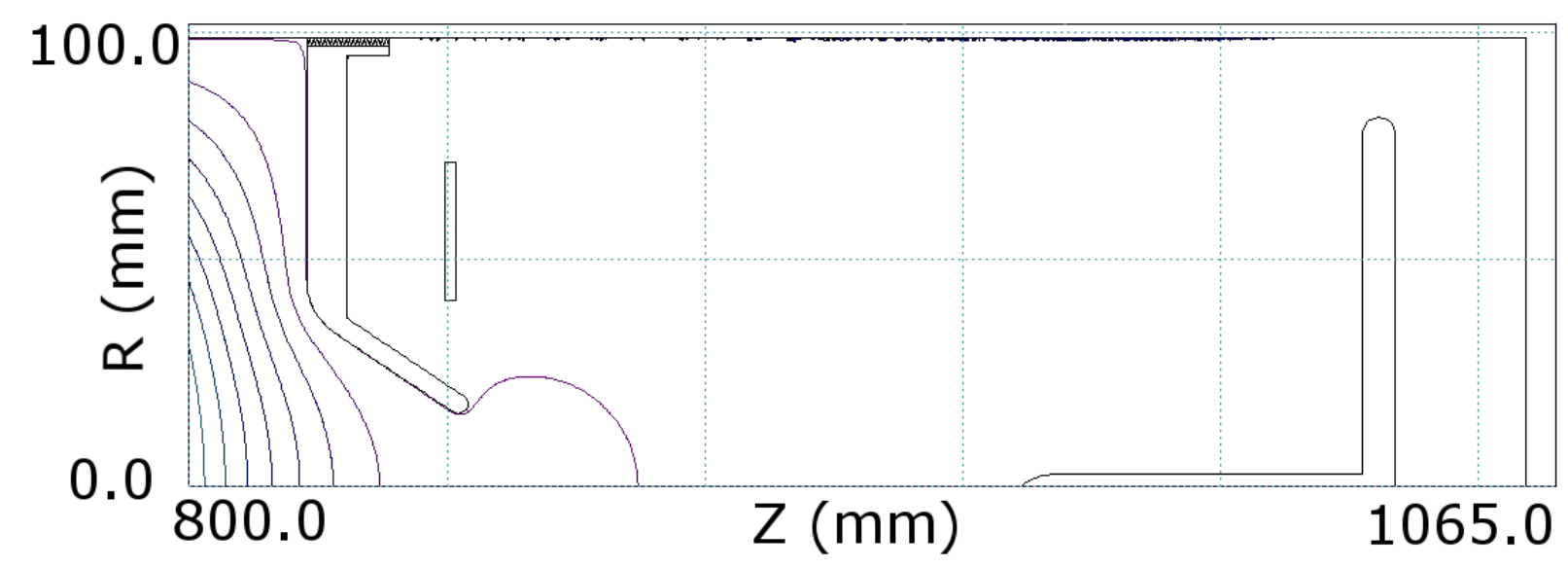

Fig. 7. Simulated trajectories of secondary electrons. $U_{\text {cath }}=250 \mathrm{kV}, \mathrm{U}_{\text {col }}=15 \mathrm{kV}, \mathrm{U}_{\text {refl }}=-5 \mathrm{kV}$.

The trajectories of the secondary electrons are barely visible because they are very short. Their propagation within EC volume effectively is inhibited by the radial electric field generated by the suppressing ring and the electron reflector. None of 500 generated particles escaped through the entrance diaphragm.

\section{CONCLUSION}

We presented a design for a depressed electron collector for a XHV vacuum-polarized electron gun. The design allows us to use a low-power HV power supply, so reducing power dissipation in the beam dump, and thus meeting allowing for the requirement for an extremely good vacuum. The energy of the primary electrons hitting the surface of the electron collector is lowered almost to the electron energy's spread. With a collector voltage higher than the primary electron's energy spread, the returned primary electron current does not exceed $0.1 \%$ of the primary electron beam's current. The escape of the secondary- and backscattered electrons into the beam pipe virtually is eliminated. The design of EC with a small entrance aperture and autonomous NEG pump assures an efficient vacuum separation of the electron collector from the transport line and the electron gun chamber.

\section{REFERENCES}

[1] V. Ptitsyn, E. Aschenauer, J. Beebe-Wang, et. al, High luminosity electron-hadron collider eRHIC, PAC-2011, TUOAN2, 2011 
[2] “The Electron Ion Collider", A white paper summarizing the scientific opportunities and the preliminary detector and accelerator design options, February 2002, http://www.phenix.bnl.gov/WWW/publish/abhay/Home_of_EIC/Whitepaper/Final/

[3] X. Chang, I. Ben-Zvi, J. Kewisch, V. Litvinenko, A. Pikin, V. Ptitsyn, A multiple cathode gun design for the eRHIC polarized electron source, BNL-94091-2011-CP

[4] V. N. Litvinenko. Gatling gun: High average polarized current injector for eRHIC. CA/AP/\#417, Jan. 2011

[5] J. Kewisch, X. Y. Chang, I. Ben-Zvi, V. Litvinenko, T. Rao, J. Skaritka, B. Sheehy, A. Pikin, W. Meng, Q. Wu, D. Pate, A. Burrill, T. Xin, Polarized electron gun development at the Brookhaven National Laboratory, J. Physics: Conference Series, 298 (2011) 012004.

[6] I. Ben-Zvi, X. Chang, V. Litvinenko, W. Meng, A. Pikin, and J. Skaritka, Generating highfrequency, rotating magnetic fields with low harmonic content, Phys. Rev. ST Accel. Beams 14, $092001(2011)$

[7] http://www.fieldp.com/ 